\title{
El prestigio ocupacional en "La tienda de ropa más grande de México". Elementos para la estimación del Capital Social en Uriangato, Guanajuato
}

\author{
Occupational Prestige in "The largest apparel store in México". \\ Elements for estimating Social Capital in Uriangato, \\ Guanajuato
}

\author{
Alejandro García \\ Universidad Autónoma de Barcelona (ESPAÑA) \\ agarciam@gmail.com \\ Octavio Martín Maza \\ Universidad Autónoma de Aguascalientes (MÉXICO)
}

Recibido: 16.01 .2012

Aceptado: 21.03.2013

\section{RESUMEN}

El artículo documenta el proceso de definición de la jerarquía de prestigio en una muestra de ocupaciones, componente necesario de un Generador de Posiciones para estimar el Capital Social en un contexto local atípico, desde una perspectiva relacional. Describe los resultados de un estudio de diseño mixto efectuado para conocer la percepción de prestigio ocupacional en Uriangato, una localidad mexicana altamente especializada en la producción y venta de ropa a través de pequeños negocios.

El estudio de caso evidencia una interpretación diferenciada del prestigio ocupacional respecto a los estándares internacionales. Las ocupaciones características de la configuración productiva especializada local son en general mejor evaluadas. Ser propietario de un negocio de manufactura, comercio o servicios, es una ocupación más reconocida en comparación con otras que, en contextos típicos, poseen mayor prestigio y estatus socioeconómico, como las de abogado, profesor o enfermera. 
No obstante, la escala obtenida con base en la técnica cualitativa de pile sort, correlaciona fuertemente con dos escalas de prestigio ocupacional internacionalmente estandarizadas, lo que posibilita construir un Generador de Posiciones localmente pertinente para realizar mediciones de Capital Social que mantengan su comparabilidad con otras realizadas en contextos económicos o culturales diferentes.

\title{
PALABRAS CLAVE
}

Capital Social. Prestigio Ocupacional. Generador de Posiciones. Especialización local.

\begin{abstract}
The article documents the process of defining the prestige hierarchy for a sample of occupations, a required component of a Position Generator to estimate Social Capital within a local atypical context, from a relational perspective. It describes the results of a mixed-design study conducted to assessing the perceptions of Occupational Prestige in Uriangato, a Mexican town highly specialized in producing and selling clothing through small businesses.

Through the case study we found a differentiated interpretation of occupational prestige regarding international standards. Occupations related to productive specialized local settings, are generally better evaluated. Owning a business of manufacturing, trade or services, is a better recognized occupation in comparison with others that, in typical contexts, have greater prestige and socioeconomic status, such as lawyer, teacher or nurse.

However, the scale achieved on the basis of the qualitative technique of pile sort, strongly correlates with two Internationally Standardized Scales of Occupational Prestige, making it possible to build a locally relevant Position Generator to measure social capital, while maintaining comparability to other studies done in different economic or cultural contexts.
\end{abstract}

\section{KEYWORDS}

Social Capital. Occupational Prestige. Position Generator. Local Specialization.

\section{EL CAPITAL SOCIAL DESDE UNA PERSPECTIVA RETICULAR}

El concepto de Capital Social (CS) ha cobrado popularidad en años recientes para el estudio de diferentes aspectos de la realidad social en múltiples escenarios y desde distintos niveles de análisis. Ha trascendido las fronteras del ámbito 
académico y es ampliamente utilizado por el público y los tomadores de decisiones en todo el mundo (Torche y Valenzuela 2011), de tal modo que podría considerarse como una de las más exitosas «exportaciones» de la sociología hacia otras ciencias sociales y al discurso público (Portes 2000), independientemente de que ha sido definido y operacionalizado de diversas maneras.

En términos de Brunie (2009), pueden identificarse al menos tres grandes enfoques para definir el Capital Social. El enfoque colectivo lo entiende como una propiedad de los grupos, cuya función es la de facilitar la coordinación y la cooperación para el beneficio mutuo y en la cual resultan fundamentales la confianza, las normas de reciprocidad, las redes, las reglas y los valores compartidos. Por otra parte, el enfoque generalista lo interpreta como una propiedad subjetiva de los individuos, referente a los valores y actitudes que influyen en la forma en que las personas se relacionan con otras y las predisponen a cooperar, confiar y desarrollar empatía con el resto. Finalmente, el enfoque relacional lo entiende como una propiedad de las redes de los actores sociales, materializada en los recursos disponibles en su entorno interpersonal, que es producto de una inversión intencional en relaciones con los demás y que puede ser usado para obtener beneficios instrumentales y expresivos.

Fulkerson y Thompson (2008) identifican dos campos en la conceptualización del CS, que si bien están en competencia, no se autoexcluyen pues existen solapamientos entre ellos. Por una parte está la comprensión normativa, asociada con las tradiciones Durkheimiana y el utilitarismo racional Weberiano, representada en los trabajos de Putnam, Coleman y Hanifan, donde el CS es visto como una explicación universal para patrones de desarrollo, que pueden entenderse en términos de niveles variantes de confianza y reciprocidad, cohesión y solidaridad, u otros aspectos que conduzcan a la acción colectiva en comunidades, regiones e incluso Estados Nación.

En el otro extremo está la visión de recursos, que partiría de las tradiciones del interaccionismo, especialmente de Simmel; y del conflicto, en la visión de Marx. En ella tendrían relevancia los trabajos de Bourdieu y de Granovetter. Aquí el CS es útil como una explicación para patrones irregulares en la acumulación de poder, prestigio y otras formas de desigualdad. Es resultado de las inversiones que los individuos realizan en sus redes de relaciones con la expectativa de algún tipo de retorno futuro, y es susceptible de convertirse en, o intercambiarse por, otros tipos de capital.

Quizá el traslape más importante entre las diferentes concepciones del Capital Social consiste en el consenso generalizado en diferentes autores como Bordieu, Coleman, Portes, Putnam, Burt y otros, en la premisa general de que el CS se origina y radica en las redes sociales de las personas (Lin y Erickson 2008; Bekkers, Völker, Van der Gaag y Flap 2008).

Nosotros argumentamos que una perspectiva del Capital Social basada en Redes Sociales, es decir reticular, implica simultáneamente las nociones de relaciones y de recursos. Para Flap y Boxman (2001) las redes sociales son capital social porque son instrumentales en el logro de metas, por ejemplo en obtener un 
trabajo. Así, el CS es resultado del tamaño de la red, la estructura de la red, las inversiones en los miembros de la red, y los recursos que poseen estos actores.

En congruencia con este argumento, el CS necesita de un soporte relacional, pues a diferencia del capital físico, no puede ser poseído por ningún actor, ni tampoco internalizado, como el capital humano, porque es la relación de un actor con otros la que contiene y transfiere los recursos poseídos por éstos (Bian 2008). Los actores individuales y sus relaciones, expresadas en las redes sociales y por tanto en la estructura mesoespacial, forman las bases del CS. Estas relaciones tienen microconsecuencias para el individuo, así como macroconsecuencias para la colectividad (Lin y Erickson 2008).

Así, para las aproximaciones colectivas - normativas, los indicadores para la estimación del CS radican, como en Putnam (2000), en aspectos tales como la vida asociativa de la comunidad, el grado de compromiso ciudadano en los asuntos públicos, el nivel de participación en acciones de voluntariado, la sociabilidad informal y la confianza. En cambio, la aproximación relacional y de recursos adoptada en este artículo coincide con la postura de Burt (2000) que propone enfocarse en los mecanismos reticulares específicos responsables de los efectos del CS, en lugar de tratar de integrar diferentes posturas en metáforas ligadas de manera imprecisa a indicadores empíricos distantes.

Esto es, que las propiedades colectivas como la confianza, instituciones, normas y valores no serían parte de la definición de CS aquí utilizada, sino que se les considera factores externos que pueden influenciar o probablemente tener un efecto en el CS (Huber 2009). Así entonces, nos apegamos a la sencilla definición de Nan Lin: El capital social son recursos incrustados en redes sociales (Lin 2001).

\section{MEDICIÓN DEL CAPITAL SOCIAL. ELICITACIÓN DE RELACIONES Y RECURSOS A TRAVÉS DEL GENERADOR DE POSICIONES}

El Análisis de Redes Sociales (ARS) como teoría y método permite aproximarse al estudio del CS, que conforme a Bian (2008) puede ser observado al menos en tres dimensiones: (i) en los lazos de red, bajo el supuesto de que a mayor disponibilidad de ligas con otros actores, mayor la posibilidad de acceder a su capital social; (ii) en la estructura de las redes, que a través de características más complejas como la densidad de la red, la posición de los actores en la estructura y sus roles, o la fortaleza o debilidad de sus lazos, significaría accesos diferenciados al capital social; y finalmente (iii) en los recursos incrustados en las redes sociales, que un actor puede obtener a través de las relaciones con sus alteri, sean directas o indirectas.

Las dos primeras dimensiones han sido las más frecuentemente estudiadas por el ARS (Van der Gaag 2005) de manera tal que se dispone de un sólido conjunto de indicadores con base en la composición y estructura de las redes, que formalizan la noción de CS tanto a nivel individual como de grupos (Borgatti, Jones y Everett 1998). 
Desde la óptica de recursos, las redes sociales y el capital social no son términos intercambiables, sino que las características y propiedades de la red deben ser interpretadas como antecedentes exógenos importantes y necesarios para el CS (Lin 2008). Así, los indicadores de medida del CS se concentran en el acceso diferenciado de los actores a los recursos sociales susceptibles de ser movilizados, considerando simultáneamente la posición del actor individual (ego) en la jerarquía social, los atributos y posición estructural de aquellos con los que se relaciona (alteri), así como la naturaleza de estas relaciones y, eventualmente, sus patrones de configuración reticular.

Citando a Van der Gaag, Snijders y Flap (2008), para analizar y dimensionar el CS comúnmente se utilizan tres tipos de instrumentos: a) El generador/ interpretador de nombres (GN), que es el de más antigua data y el más aplicado por muchos investigadores en la tradición del ARS, dado que proporciona los elementos para el análisis de la composición y estructura de las redes sociales, al obtener información sobre las relaciones de un actor con otros y sus características; b) el generador de recursos (GR), desarrollado por Snijders (1999) y Van der Gaag y Snijders (2005), que consiste en inquirir a los actores sobre sus posibilidades reales o potenciales de acceder a una serie predefinida de recursos, ya sean de naturaleza instrumental o expresiva, a través de sus relaciones personales; y c) el generador de posiciones (GP), propuesto originalmente por Lin y Dumin (1986), en que explícitamente nos enfocamos en este artículo.

El GP es el método de medición que, con diversas variantes y adaptaciones contextuales, ha sido empleado por diversos autores que suscriben como definición común de CS, la ya citada de recursos incrustados en redes sociales (Lin y Erickson, 2008). El método incluye indicadores sobre tres aspectos esenciales para medirlo: La presencia de alteri, los recursos de estos alteri, y la disponibilidad de dichos recursos para ego. Los datos obtenidos son indicativos del acceso a los recursos disponibles en las redes de los sujetos, su CS potencialmente utilizable (Van der Gaag et al. 2008).

Los GP utilizan una muestra de posiciones estructurales, normalmente ocupaciones, para determinar el acceso desigual de las personas a diferentes niveles de la estructura social, a través de sus contactos. En términos de Lin y Erickson (2008), dado el rol clave del trabajo en las sociedades modernas, las ocupaciones serían las primeras locaciones sociales, aunque no las únicas, en las que podría identificarse una jerarquía de posiciones sociales, a partir del prestigio que tienen en la sociedad, indicador que a su vez estaría estrechamente ligado a diferencias en recursos disponibles para sus ocupantes.

Esta muestra de ocupaciones normalmente se obtiene de las clasificaciones nacionales correspondientes y representa un rango escalado de valores numéricos conforme a la puntuación de cada ocupación en una determinada escala de estatus o prestigio ocupacional. Los informantes son requeridos para indicar si conocen o tienen relación con al menos a una persona que desempeñe cada una de las ocupaciones. Las respuestas positivas asumen el valor del prestigio ocupacional que corresponda. 
Con estos datos es posible construir medidas en relación a 1) el rango de accesibilidad a diferentes posiciones jerárquicas; 2) la extensión o heterogeneidad de la accesibilidad a esas posiciones; y 3 ) la alcanzabilidad superior del CS accedido (Lin, Fu y Hsung 2001). En términos generales, lo que se tiene son indicadores acerca de la presencia de elementos específicos en la red social, el volumen y la diversidad de la misma, a partir de diferentes cálculos con los datos obtenidos (Van der Gaag et al. 2008).

Adicionalmente, también pueden obtenerse otros datos tanto de índole relacional como atributiva, tales como el tipo de relación entre ego y alteri, su fortaleza y frecuencia, entre otros. Entonces, a diferencia de un GN que obtiene información relacional de contenido o de roles, el GP la obtiene sobre la relación con los ocupantes de posiciones jerárquicas (Lin 2001).

\section{CUESTIONES METODOLÓGICAS. DE LA ESTANDARIZACIÓN EN LAS SOCIEDADES MODERNAS COMPLEJAS A LA ADAPTACIÓN PARA CONTEXTOS LOCALES ATÍPICOS}

Dos elementos constitutivos del GP plantean interesantes cuestiones metodológicas y se convierten en el centro de la discusión sobre si es posible utilizar el GP como un instrumento fiable de medición del CS en todo tipo de contextos y en todo caso, cuáles son las adaptaciones que deben hacerse de acuerdo a las condiciones específicas de las localidades o poblaciones a estudiar.

El primero es la obtención y el tratamiento de las ocupaciones que constituyen el listado integrado en el GP, a partir del cual se pretende representar los diferentes niveles de la estructura social, como reflejo de la ocupacional. Por ejemplo ¿es un mismo grupo de ocupaciones pertinente en dos contextos socioculturales distintos? ¿Significa lo mismo una actividad laboral determinada en comunidades o países diferentes?

Existe un alto nivel de estandarización en las clasificaciones de ocupaciones. Una de las más aceptadas en la actualidad es la Clasificación Internacional Uniforme de Ocupaciones (ISCO, por sus siglas en inglés), generada por la Organización Internacional del Trabajo, que sirve de modelo para una gran cantidad de clasificaciones a nivel nacional e incluso es aplicada en países que no disponen de las propias, y por lo tanto permite reportar datos uniformes y realizar comparaciones internacionales con un aceptable nivel de precisión. Aunque su versión más reciente es la ISCO-08, la que más extensamente continúa utilizándose a la fecha es la ISCO-88 (ILO 2011).

Para el caso mexicano, el Instituto Nacional de Estadística y Geografía (INEGI) ha desarrollado la Clasificación Mexicana de Ocupaciones (CMO), que si bien toma como modelo la ISCO-88, a partir de las experiencias censales también incluye ocupaciones más propias del complejo contexto nacional, especialmente al reconocer la presencia de fenómenos como la extendida informalidad. No obstante, mantiene la comparabilidad nacional e internacional (INEGI 2009a). 
La tendencia reciente es que en el GP se incluyan ocupaciones que reflejen adecuadamente la jerarquía ocupacional de la comunidad o sociedad que se estudia (Lin y Erickson 2008) con el fin de adaptarlo a contextos específicos, es decir, asegurar que la muestra de ocupaciones provenga de una jerarquía «significativa» (Lin 2001) y que se garantice la presencia de rangos claramente diferenciados en la escala. Nosotros argumentamos, junto a otros autores (Bian 2008; Enns, Malinick y Matthews 2008), que obviar la introducción de actividades localmente pertinentes o la percepción específica de su prestigio, podría inducir matices equivocados en la medición del CS.

El segundo elemento importante es la determinación de la escala o escalas de estatus o prestigio ocupacional utilizadas para valuar y jerarquizar las ocupaciones de la muestra, sobre las cuales se basarán los cálculos de las medidas del CS. Es decir ¿Las ocupaciones tienen niveles de prestigio similares en dos sociedades o culturas diferentes?

El nivel de estandarización en este sentido también es elevado. Entre los diversos esquemas de estratificación ampliamente conocidos pueden citarse la propia ISCO, estructurada de acuerdo al tipo de tareas y las habilidades o nivel educativo requeridos para desempeñarlas; la escala de Goldthorpe y Erickson o EGP, que ofrece una agrupación alrededor de clases; la de estructura social de Eric Olin Wrigt, centrada en las relaciones laborales; la de ocupación francesa; las categorías socioprofesionales de Suiza (CSP-CH); la escala de interacción y estratificación social de Cambridge (CAMSIS); la internacional estandarizada de prestigio ocupacional de Treiman (SIOPS); y el índice socioeconómico internacional de estatus ocupacional de Ganzeboom y Treiman (ISEI) (Barozet 2007; Bergman y Joye 2001).

Estas dos últimas escalas, la SIOPS y el ISEI, que en este artículo denominamos genéricamente como Escalas Internacionalmente Estandarizadas (EIE), son quizá las más ampliamente difundidas puesto que han sido utilizadas en múltiples estudios de capital social (Flap y Völker 2008; Lin y Ao 2008) e incluso en contextos transnacionales (Chen y Tan 2009); también como base para desarrollar escalas de prestigio nacionales, como por ejemplo la PRESCA para el caso español (Carabaña y Gómez 1996) y que además han sido ampliamente compatibilizadas con la ISCO-88 (Ganzeboom y Treiman 1996).

Ambas escalas tienen diferencias entre ellas, pues mientras que los índices socioeconómicos se refieren más directamente a recursos humanos y recompensas económicas, las medidas de prestigio se refieren más a «recompensas sociales que las personas pueden esperar en interacciones humanas» (Ganzeboom y Treiman 2003, citados en Van der Gaag et al. 2008). Así, el ISEI es un índice socioeconómico que puntúa las ocupaciones de acuerdo a sus niveles promedio de educación e ingreso a partir de datos censales y de encuestas, mientras que la SIOPS sintetiza medidas de prestigio a partir de evaluaciones populares sobre el posicionamiento de una ocupación. No obstante, ambas escalas están fuertemente correlacionadas (Bergman y Joye 2001; Ganzeboom y Treiman 1996).

Nuestros cuestionamientos emergen del trabajo actual de investigación que realizamos sobre la precariedad laboral y el capital social en contextos locales 
atípicos, específicamente en localidades de alta especialización productiva en el centro occidente de México, que implica el diseño y la aplicación de un GP. Para la selección de la muestra de ocupaciones nos basamos en la ya descrita CMO; sin embargo, en México no se dispone de una escala nacional de prestigio ocupacional que pudiera utilizarse como criterio de estratificación.

En principio, una solución teóricamente factible consistió en recurrir a la utilización de las EIE, sustentados en las afirmaciones de Treiman acerca de que todas las «sociedades modernas complejas» estarían organizadas en funciones societales similares, incluyendo una división del trabajo y una percepción subjetiva del prestigio ocupacional también muy similares. Estos supuestos provienen de sus hallazgos a partir del análisis de 85 estudios en 60 contextos diferentes, a partir de los cuales construyó la escala SIOPS de 1977 y entre los que encuentra una correlación de $\mathrm{r}=0.79$ y s $=0.14$, misma que considera suficiente para rechazar los argumentos acerca de las influencias culturales de contextos específicos sobre la percepción de la estructura ocupacional (Bergman y Joye 2001).

Sin embargo, la evidencia sobre el carácter atípico de las comunidades que estudiamos, nos condujo a preguntarnos: ¿qué ocurre con estas aseveraciones, en localidades cuya lógica organizativa y productiva no es equiparable a las de las «sociedades modernas complejas»? ¿Continúan siendo válidas para espacios locales cuya configuración ocupacional difiere de la tradicional o clásica? ¿Hasta dónde hay correspondencia entre las EIE con la interpretación del prestigio ocupacional local, especialmente en contextos atípicos?

\section{UN CASO DE ESTUDIO. LAS ESPECIFICIDADES LOCALES DE URIANGATO, GUANAJUATO, MÉXICO}

Por contextos atípicos nos referimos a una serie de localidades productivamente especializadas en la República Mexicana, en las que actualmente se realizan diversos proyectos de investigación, donde se ha identificado la extendida presencia de una serie de características y factores peculiares.

i. Procesos de industrialización atípicos, en los que el desarrollo se ha dado a partir de iniciativas naturales locales, normalmente sin intervención de una política pública deliberada. Se trata de localidades que combinan lo rural con lo urbano, a partir de micro, pequeñas y medianas empresas, sobre todo talleres de corte familiar, casi siempre con alta concentración en torno a una actividad productiva y en las que coexisten procesos artesanales con otros tecnológicamente avanzados. Estas localidades no necesariamente comparten las características del Distrito Industrial ideal (Lozares, Molina, García Macías y Maza 2011; Maza, García Macías y Ortega 2011).

ii. Trabajo Precario, entendido como aquél que presente una o más características en oposición al trabajo típico ideal. Nos referimos al trabajo inestable, sin contrato o seguridad social y no necesariamente de 
tiempo completo; no siempre industrial ni en un espacio fijo, sino que también puede darse en otros espacios, a veces incluso en los reproductivos; no subordinado a un patrón o bien estándolo a más de uno; sin pertenecer a una empresa o trabajando para más de una; con actividades mixtas en el mercado de trabajo; empleo no asalariado, precario o arriesgado; trabajo en la informalidad o ilegalidad, etc. Estos modelos implican, contrario a los modelos tayloristas-fordistas, la combinación de espacios y de lógicas (lo productivo-reproductivo, la ganancia-los afectos) (De la Garza 2010; Neffa, Olivera, Persia y Trucco 2010).

iii. Estructura ocupacional especializada. Estas comunidades tienen una elevada concentración del mercado de trabajo en torno a actividades industriales o terciarias similares, dando lugar a procesos peculiares de configuración social de la ocupación, con trayectorias laborales y jerarquías ocupacionales propias que los caracterizan y que rompen con el modelo del trabajo asalariado (Lozares et al. 2011).

iv. Desarrollo económico regionalmente diferenciado, pues son localidades en las que, pese a su carácter altamente informal y precario, se tienen mejores niveles de desarrollo humano y presentan menos pobreza, marginación, rezago social y desigualdad que otras ciudades o regiones geográficamente próximas. En ellas se resuelvan las necesidades de seguridad y certidumbre mediante formas cercanas a las estrategias de sobrevivencia, aunque no siempre están vinculadas a la pobreza (García Macías 2011).

Este marco contextual permite introducir elementos para analizar el caso específico de Uriangato. Se trata de un municipio del estado de Guanajuato, en el centro occidente de México, que al igual que Moroleón, municipio conurbado, es altamente especializado en la producción y venta de ropa. La ciudad se promueve oficialmente como «La tienda de ropa más grande de México ${ }^{1} »$.

Según datos de los Censos Económicos 2009, en las actividades relacionadas con la industria de la ropa trabajan cuatro de cada diez personas ocupadas. Los negocios dedicados a la fabricación de textiles, prendas de vestir e insumos relativos (617) representan el 64\% de las unidades económicas y concentran al 73\% del Personal Ocupado (PO) de la Industria Manufacturera (IM). Hay además 1218 comercios de ropa al por menor, uno por cada 48 habitantes (INEGI 2009b).

Las empresas manufactureras y los comercios son fundamentalmente negocios familiares, cuyo tamaño promedio es de tres y dos personas respectivamente. El personal que trabaja sin pago (Propietarios, familiares y otros trabajadores no remunerados) representa el 53\% de la IM y el 66\% del comercio al por menor (INEGI 2009b). Este indicador muestra que existe un alto índice de trabajadores que son propietarios de los medios de producción.

${ }^{1}$ http://www.uriangato.gob.mx/conocenos/info.php 
Las remuneraciones anuales por persona en la producción de ropa rondan los 8 dólares al día (36 mil pesos anuales) y en el comercio de ropa al por menor, 6.7 dólares (INEGI 2009b). Es importante mencionar que Uriangato funciona en articulación con su vecino Moroleón y con localidades como Zapotlanejo y Villa Hidalgo, en el estado de Jalisco. Uriangato se especializa en la comercialización de las prendas de vestir, lo que define el perfil de la mano de obra.

Tabla 1. Relación Personal Ocupado sobre Unidades Económicas para sectores elegidos, Estado de Guanajuato y Municipio de Uriangato, 2009

\begin{tabular}{ccccc}
\hline \multirow{2}{*}{ Estado/Municipio } & Sector & $\begin{array}{c}\text { Unidades } \\
\text { Económicas } \\
(\mathrm{UE})\end{array}$ & $\begin{array}{c}\text { Personal } \\
\text { Ocupado } \\
\text { Total (POT) }\end{array}$ & $\begin{array}{c}\text { Relación } \\
\text { POT/UE }\end{array}$ \\
\hline Guanajuato & Industria Manufacturera & 23112 & 269609 & 11.6 \\
Guanajuato & Comercio al por mayor & 6523 & 51154 & 7.8 \\
Guanajuato & Comercio al por menor & 85547 & 238899 & 2.7 \\
\hline Uriangato & Industria Manufacturera & 965 & 3052 & 3.1 \\
Uriangato & Comercio al por mayor & 92 & 628 & 6.8 \\
Uriangato & Comercio al por menor & 2311 & 5519 & 2.3 \\
\hline
\end{tabular}

INEGI. Censos Económicos 2009. Resultados Definitivos

El desempleo, sin embargo, se mantiene en tasas menores a las de la región. Según el Censo de Población y vivienda 2010, mientras que el $6.57 \%$ de los hombres económicamente activos de Uriangato se encontraban desocupados, en el caso de las mujeres la cifra se reduce al $1.95 \%$. Esto indica que en la industria de la ropa continúa existiendo un predominio laboral de las mujeres sobre los hombres. El total de población desocupada con relación a la Población Económicamente Activa es de $4.93 \%$, inferior al 5.47 del estado de Guanajuato (INEGI 2010).

No obstante que se trata de micronegocios con estructuras atípicas, su productividad no es baja. Como se muestra en la tabla2, la productividad de los trabajadores de Uriangato está por encima de la nacional.

Tabla 2. Producción Bruta por trabajador para sectores escogidos, República Mexicana y Uriangato, 2009

\begin{tabular}{lcccc}
\hline País/Municipio & Sector & $\begin{array}{c}\text { Producción Bruta } \\
\text { Total (PBT) } \\
\text { (Miles de Pesos) }\end{array}$ & $\begin{array}{c}\text { Personal } \\
\text { Ocupado } \\
\text { Total (POT) }\end{array}$ & $\begin{array}{c}\text { (PBT/POT) } \\
\text { (Miles de Pesos } \\
\text { porabajador) }\end{array}$ \\
\hline $\begin{array}{c}\text { Estados Unidos } \\
\text { Mexicanos } \\
\text { Uriangato }\end{array}$ & Manufactura y Comercio & $248,952,024$ & $3,100,941$ & 80.28 \\
\hline
\end{tabular}

INEGI. Censos Económicos 2009. Resultados Definitivos 
Los indicadores del desarrollo económico atípico de Uriangato son numerosos. Como ejemplo en este artículo, en la tabla 3 se presenta el Coeficiente de Gini, uno de los indicadores más utilizados para evaluar la desigualdad en la población -cuyos valores se expresan de 0 a 1,-entre más cercano al 1 se puede decir que existe mayor desigualdad en la distribución en el ingreso en una población determinada- en el que tanto para el año 2000 como para el 2005, sus valores están por debajo del estatal y del nacional, además con una significativa reducción en ese lapso, al pasar de 0.4324 a 0.3667 , lo que supone un descenso en las desigualdades por ingreso.

Tabla 3. Indicadores de Desigualdad, Nacional, Estado de Guanajuato y Municipio de Uriangato, 2000 y 2005

\begin{tabular}{|c|c|c|c|c|c|c|}
\hline País/Estado & $\begin{array}{l}\text { Coeficiente } \\
\text { de Gini } \\
2000\end{array}$ & $\begin{array}{l}\text { Razón de } \\
\text { ingreso del } \\
10 \% \text { más } \\
\text { rico entre } \\
\text { el } 10 \% \\
\text { más pobre }\end{array}$ & $\begin{array}{l}\text { Razón de } \\
\text { ingreso del } \\
5 \% \text { más } \\
\text { rico entre } \\
\text { el } 5 \% \text { más } \\
\text { pobre }\end{array}$ & $\begin{array}{l}\text { Coeficiente } \\
\text { de Gini } \\
2005\end{array}$ & $\begin{array}{l}\text { Razón de } \\
\text { ingreso del } \\
10 \% \text { más } \\
\text { rico entre } \\
\text { el } 10 \% \\
\text { más pobre }\end{array}$ & $\begin{array}{l}\text { Razón de } \\
\text { ingreso del } \\
5 \% \text { más } \\
\text { rico entre } \\
\text { el } 5 \% \text { más } \\
\text { pobre }\end{array}$ \\
\hline Nacional & 0.5458 & 42.3 & 83.2 & 0.5006 & 27.7 & 52.7 \\
\hline Guanajuato & 0.5254 & 33.4 & 64.3 & 0.4820 & 24.8 & 46.7 \\
\hline Uriangato & 0.4324 & 17.0 & 30.0 & 0.3667 & 10.6 & 17.6 \\
\hline
\end{tabular}

CON EVAL. Indicadores de Desigualdad con errores estándares, 2000 y 2005.

http://www.coneval.gob.mx/crosconeval/rw/pages/index.es.do

Asimismo, las razones de ingreso, que miden las proporciones de ingreso entre el $10 \%$ y $5 \%$ más rico de la población y el $10 \%$ y $5 \%$ más pobre respectivamente, muestran que las disparidades de ingreso entre ricos y pobres son mucho menores a las de la media nacional y estatal. Para 2005 la desigualdad a nivel nacional era tres veces mayor que la de Uriangato. Estos datos demuestran que la riqueza está menos concentrada, lo que nos hace suponer que los estratos sociales y por tanto el capital social pueden también ser peculiares.

\section{DISEÑO DE UN ESTUDIO DE MÉTODO MIXTO \\ PARA CONOCER LA PERCEPCIÓN LOCAL ACERCA DEL PRESTIGIO OCUPACIONAL}

Con el fin de validar/adaptar nuestro GP, realizamos un estudio cuyos objetivos consistieron en verificar, mediante un diseño mixto, a) hasta dónde una serie de ocupaciones resultaba significativa para los habitantes de una localidad de configuración ocupacional atípica, Uriangato, en términos de conocimiento de la existencia de las actividades y comprender sus denominaciones; b) La per- 
cepción local en torno a los niveles de prestigio de una serie de ocupaciones estándar; c) el comportamiento de algunas innovaciones al GP tradicional, como la inclusión de ocupaciones con prestigio negativo (o desprestigio) y de otras tareas no clasificadas en la CMO, tales como el jubilado, el ama de casa o el trabajador no asalariado en el negocio familiar; y d) la congruencia del prestigio ocupacional local percibido con las EIE, que garantizara la comparabilidad internacional de nuestros estudios de CS en la región.

El estudio fue dividido en dos segmentos, A y B, aplicando un instrumento distinto en cada uno de ellos. En ambos se solicitó información sobre un listado relativamente amplio de 30 ocupaciones. En el segmento A se trató de un listado tradicional, construido acorde a la experiencia plasmada en la literatura, que incluye actividades más estándar, además de algunas otras consideradas como pertinentes al contexto local, todas ellas incluidas tanto en la ISCO-88 como en la CMO, y con valores disponibles en las escalas SIOPS e ISEI.

En el segmento B se utilizó un listado más abierto, en el que a) se mantuvieron 16 de las ocupaciones del primero, razonablemente distribuidas entre prestigio y estatus alto, medio y bajo, aunque en cinco de ellas se modificó ligeramente la denominación, sin que perdiera su congruencia con la CMO; b) se cambiaron tres actividades por otras similares pero con la probabilidad de resultar más significativas en el contexto de la industria de la ropa: agente de ventas por proveedor de maquinaria o materia prima industrial, técnico en electricidad o electrónica por técnico o mecánico de maquinaria industrial, y chofer de carga o de pasajeros por transportista; y c) se excluyeron once ocupaciones estándar.

Para sustituir estas ocupaciones excluidas se integraron a) cuatro que se supondrían con un claro prestigio negativo: prestamista o agiotista, gestor o intermediario comercial o coyote, adivino o brujo y vendedor de mercancía pirata; b) cuatro más que consideramos reflejan adecuadamente la composición del mercado de trabajo local y que no estaban incluidas en el primer instrumento: maquilador de ropa a domicilio, ayudante de tejido o bordado, propietario arrendador de locales o fincas, y peluquero o estilista; y c) tres actividades que no se consideran propiamente ocupaciones conforme a la CMO y de las que no se dispone información de sus puntuaciones en las EIE: pensionado o jubilado, ama de casa, y trabajador no asalariado en el negocio familiar.

En ambos segmentos el procedimiento consistió en proporcionar al informante un bloque de cartas, cada una de las cuales contenía una de las 30 ocupaciones del instrumento. El diseño mixto consistió en la aplicación para cada segmento de dos ejercicios distintos de valoración de las ocupaciones: uno cualitativo y otro cuantitativo.

La fase cualitativa tuvo un enfoque basado en la antropología cognitiva, buscando representar el sistema lógico de pensamiento de la localidad vista como una cultura diferente. El supuesto es que la estructura ocupacional puede ser parte de un modelo cultural o marco interpretativo compartido por los miembros de la localidad y que el nivel de prestigio de una serie de ocupaciones puede entenderse como un dominio cultural, es decir «un conjunto de ítems del mismo 
tipo relacionados por una estructura interna de relaciones» (Borgatti 1998, en Molina y Bertrán 2008:4) que en este caso es de naturaleza jerárquica.

Por lo tanto se utilizó una técnica del tipo pile sort que consistió en solicitar al informante a) que formase dos pilas de cartas, según si conocía personalmente o no a alguien que desempeñara la ocupación correspondiente, con el fin de determinar el nivel de conocimiento y pertinencia local de las mismas; b) que ordenase el mazo de cartas en función del mayor o menor nivel de prestigio que a su juicio tenía cada ocupación en la localidad, con el fin de asignarles posteriormente un puntaje según su posición en la pila. Con este segundo procedimiento se consigue construir una escala ordinal de prestigio que, además de reflejar la idea básica de estratificación ocupacional que sustenta los presupuestos teóricos subyacentes al método del generador de posiciones, resulta susceptible de ser contrastada con otras escalas.

La segunda fase fue de tipo cuantitativo, y consistió en solicitar al informante la asignación de un puntaje numérico a cada una de las ocupaciones, conforme al prestigio que le atribuyese. Para los informantes del segmento A el rango fue de 0 a 100 y para los del segmento A de -100 a 100, con la intención de observar posibles datos de desprestigio ocupacional.

Como producto de los dos segmentos y las dos fases respectivas en cada uno, se obtuvieron cuatro variantes de lo que hemos denominado Escala de Prestigio Ocupacional Atípico (EPOA), como se detalla en la tabla 4.

Tabla 4. Variantes de la escala EPOA según el uso de diferentes listados de ocupaciones y métodos de jerarquización. Estudio sobre percepción del prestigio ocupacional en Uriangato, 2011

\begin{tabular}{|c|c|c|c|}
\hline \multirow{2}{*}{ : } & \multicolumn{3}{|c|}{$\begin{array}{l}\text { Segmento B: Con ocupaciones tradicionales, localmente pertinentes, atípicas } \\
\text { y de prestigio negativo. }\end{array}$} \\
\hline & \multicolumn{3}{|l|}{$\begin{array}{l}\text { Segmento A: Con ocupaciones tradicionales y localmente } \\
\text { pertinentes. }\end{array}$} \\
\hline \multirow{3}{*}{ 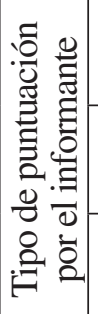 } & $\begin{array}{l}\text { Pile sort. Puntuación posterior entre } 30 \text { y } 1 \text { conforme } \\
\text { al orden de las cartas y traducción a base } 100 .\end{array}$ & EPOA al & EPOA b1 \\
\hline & $\begin{array}{l}\text { Puntuación libre en una escala de prestigio positivo } \\
\text { de } 0 \text { a } 100\end{array}$ & \multirow[t]{2}{*}{ EPOA a2 } & - \\
\hline & $\begin{array}{l}\text { Puntuación libre en una escala de prestigio - despres- } \\
\text { tigio de - } 100 \text { a } 100\end{array}$ & & EPOA b2 \\
\hline
\end{tabular}

La muestra se conformó por 23 informantes seleccionados por conveniencia, en base a perfiles determinados principalmente por su ocupación y nivel educativo. Se incluyeron informantes a) que se dedicaran a actividades no relacionadas directamente con la industria de la ropa, pero que por razón del contacto con la gente en su trabajo - empleados de gobierno, profesores o propietarios de res- 
taurante- pudieran tener una perspectiva amplia sobre el prestigio ocupacional de la población; b) trabajadores ocupados en actividades relacionadas con la confección de ropa, desde propietarios de empresas establecidas o maquiladores a domicilio, hasta profesionales y técnicos del área; c) desempleados y estudiantes no incorporados al mercado de trabajo.

Respecto a los niveles educativos, en la selección estuvieron integrados informantes cuyos niveles cubren desde la educación inicial hasta los posgrados, como se representa en la figura 1. En el proceso de búsqueda de los informantes se decidió excluir de la muestra a varias personas que cubrían el perfil ocupacional pero que no sabían leer o escribir, lo que por la naturaleza del ejercicio resultaba una limitante.

Figura 1. Escolaridad de los informantes. Estudio sobre percepción del prestigio ocupacional en Uriangato, 2011

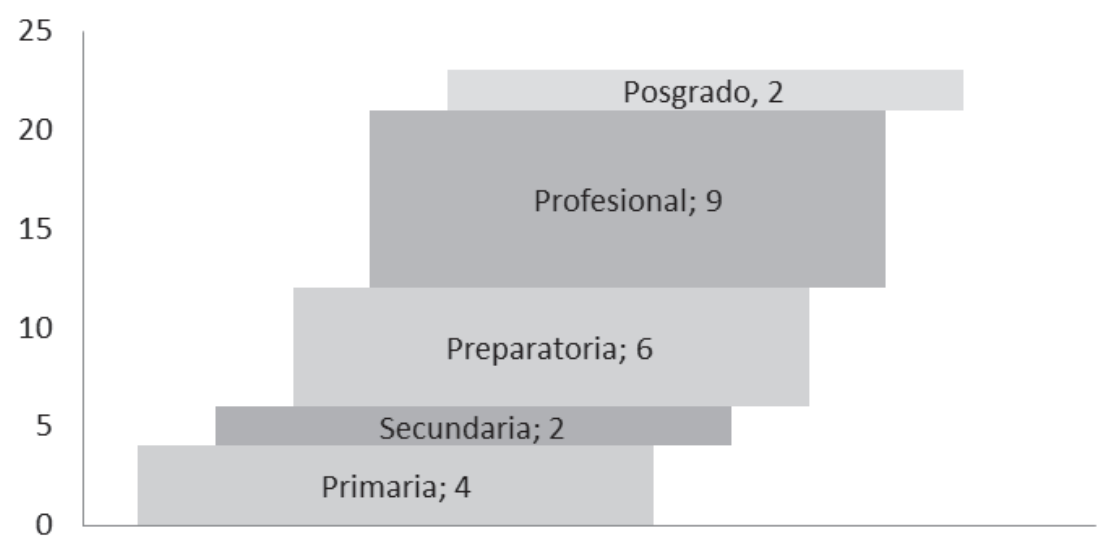

La proporción de informantes hombres entrevistados fue mayor que la de mujeres (14 y 9 respectivamente), en gran parte debido a la disponibilidad para realizar las entrevistas durante el trabajo de campo. La media de edad de los informantes fue de 30.4 años, donde el más joven tiene 18 y el más viejo 60 . La asignación del tipo de cuestionario aplicado al informante fue aleatoria.

\section{RESULTADOS. EL PRESTIGIO OCUPACIONAL LOCALMENTE DIFERENCIADO DE URIANGATO}

En principio, mediante la técnica del pile sort se comprobó que el total de las ocupaciones enlistadas en ambos segmentos, resulta significativo para la población analizada, pues los informantes, además de comprender sus denominaciones, tienen relación de conocimiento, amistad o parentesco con alguna persona que las desempeña, tal como se muestra en las figuras 2 y 3. No hay un patrón claro de correlación entre el nivel de prestigio de las ocupaciones, ya sea de las 
EIE o de nuestras propias escalas, con la cantidad de informantes que conocen a alguien que se dedique a ellas.

Figura 2. Nivel de conocimiento de las ocupaciones del segmento A. Estudio sobre percepción del prestigio ocupacional en Uriangato, 2011

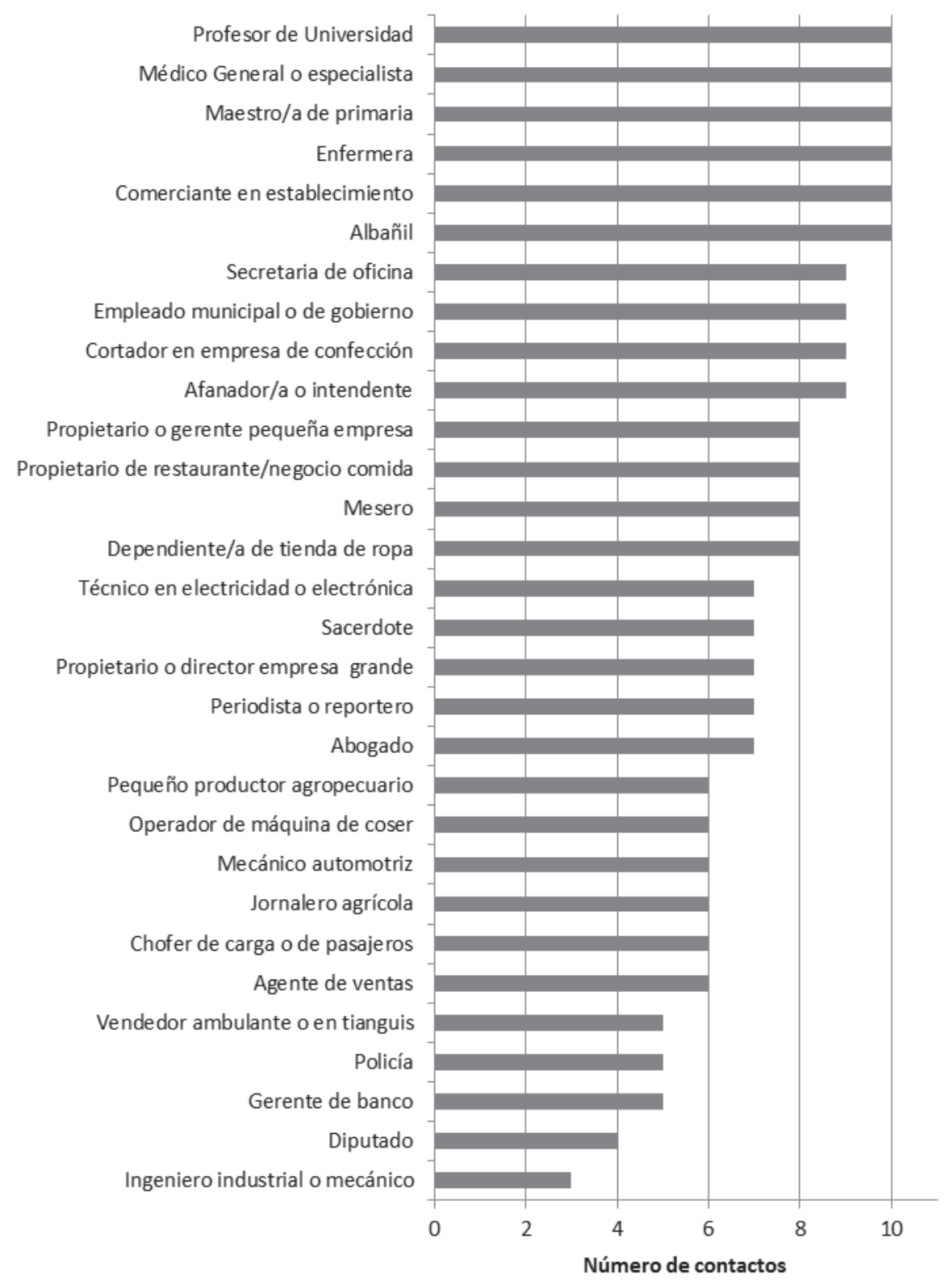

EMPIRIA. Revista de Metodología de Ciencias Sociales. N. ${ }^{\circ}$ 26, julio-diciembre, 2013, pp. 117-148. ISSN: 1139-5737, DOI: 10.5944/empiria.26.7155 
Figura 3. Nivel de conocimiento de las ocupaciones del segmento B. Estudio sobre percepción del prestigio ocupacional en Uriangato, 2011

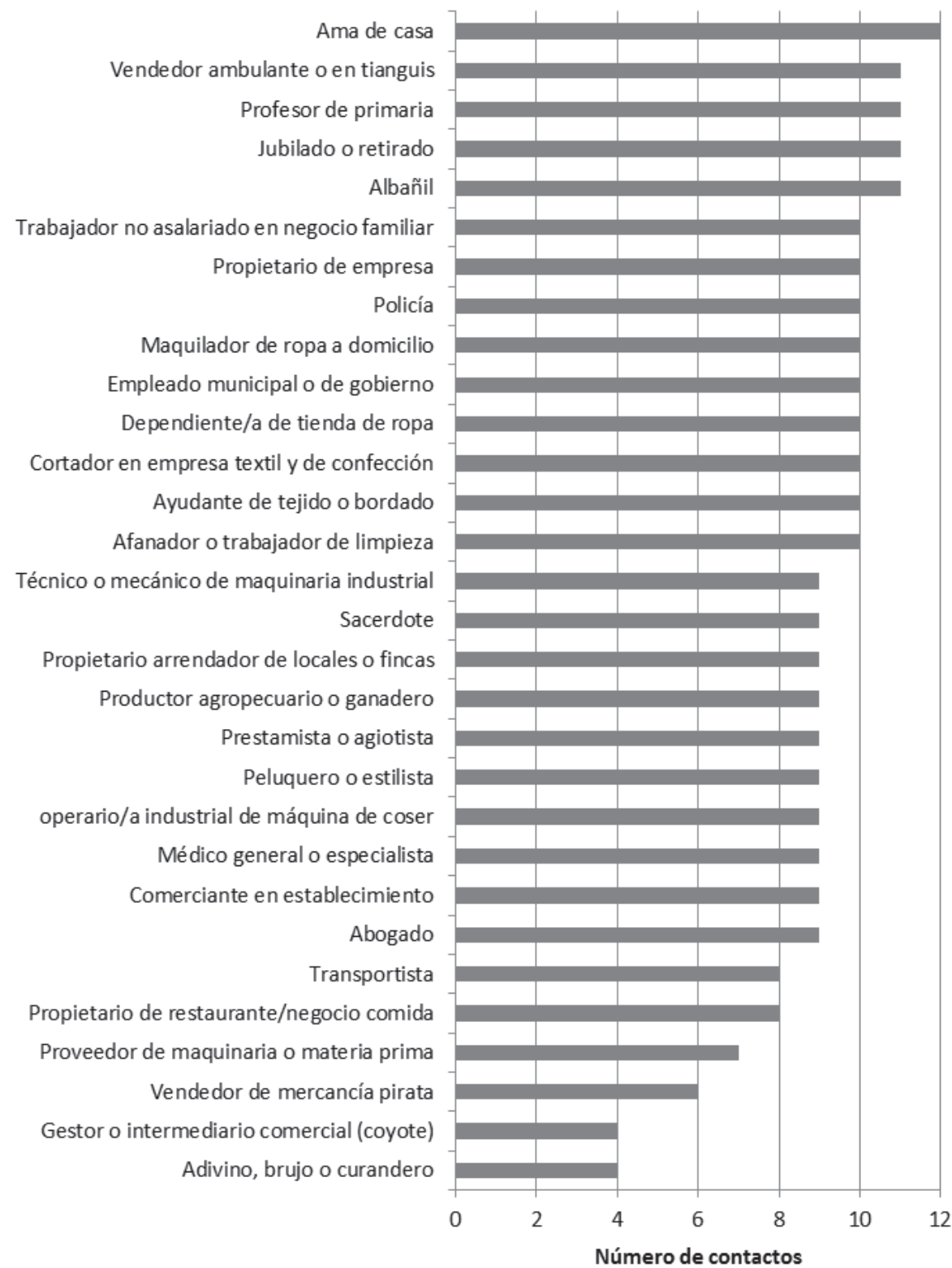

Seguidamente y con el fin de determinar el mejor procedimiento para la comprensión del prestigio ocupacional, los dos segmentos del estudio y las cua-

EMPIRIA. Revista de Metodología de Ciencias Sociales. N. ํ 26, julio-diciembre, 2013, pp. 117-148. ISSN: 1139-5737, DOI: 10.5944/empiria.26.7155 
tro variantes obtenidas de la EPOA se analizaron a la luz de su congruencia entre sí y respecto a las EIE más frecuentemente utilizadas, la SIOPS y el ISEI.

\section{Análisis del Segmento A}

La tabla 5 contiene las puntuaciones obtenidas por las 30 ocupaciones tradicionales y otras localmente pertinentes, utilizadas en este segmento del estudio.

Tabla 5. Puntuaciones de prestigio de las ocupaciones en el segmento A. Estudio sobre percepción del prestigio ocupacional en Uriangato, 2011

\begin{tabular}{lcccc}
\hline CMO OCUPACIÓN & EPOAa1 & EPOAa2 & SIOPS & ISEI \\
\hline 1401 Abogado & 62 & 63 & 73 & 73 \\
8124 Afanador/a o intendente & 20 & 47 & 21 & 16 \\
7130 Agente de ventas & 52 & 58 & 47 & 55 \\
5260 Albañil & 16 & 47 & 15 & 21 \\
4109 Chofer de carga o de pasajeros & 25 & 49 & 32 & 34 \\
1220 Comerciante en establecimiento & 62 & 65 & 46 & 49 \\
1205 Cortador en empresa de confección & 47 & 62 & 40 & 36 \\
2127 Dependiente/a de tienda de ropa & 44 & 63 & 32 & 43 \\
2121 Diputado & 69 & 65 & 64 & 77 \\
1330 Empleado municipal o de gobierno & 62 & 59 & 52 & 56 \\
6200 Enfermera & 60 & 68 & 54 & 43 \\
1180 Gerente de banco & 67 & 68 & 60 & 87 \\
1104 Ingeniero industrial o mecánico & 76 & 75 & 66 & 67 \\
7201 Jornalero agrícola & 25 & 54 & 23 & 16 \\
6290 Maestro/a de primaria & 58 & 71 & 57 & 66 \\
5520 Mecánico automotriz & 42 & 58 & 43 & 34 \\
1130 Médico General o especialista & 88 & 85 & 78 & 88 \\
8101 Mesero & 18 & 56 & 21 & 34 \\
2124 Operador de máquina de coser & 55 & 67 & 25 & 32 \\
5322 Pequeño productor agropecuario & 46 & 56 & 47 & 43 \\
5245 Periodista o reportero & 46 & 55 & 58 & 65 \\
5322 Policía & 28 & 46 & 40 & 50 \\
2100 Profesor de Universidad & 69 & 73 & 78 & 77 \\
2111 Propietario de restaurante/negocio comida & 68 & 67 & 38 & 44 \\
1300 Propietario o director empresa grande & 85 & 81 & 60 & 68 \\
1160 Propietario o gerente pequeña empresa & 83 & 72 & 50 & 51 \\
4120 Sacerdote & 52 & 61 & 60 & 53 \\
7100 Secretaria de oficina & 49 & 58 & 53 & 53 \\
8301 Técnico en electricidad o electrónica & 45 & 58 & 46 & 46 \\
7111 Vendedor ambulante o en tianguis & 38 & 56 & 23 & 25 \\
\hline
\end{tabular}

EMPIRIA. Revista de Metodología de Ciencias Sociales. N. o 26, julio-diciembre, 2013, pp. 117-148. ISSN: 1139-5737, DOI: 10.5944/empiria.26.7155 
Tal y como ocurre con su comportamiento a nivel general, los valores en las escalas SIOPS e ISEI para la serie de ocupaciones elegida, mantienen un elevado nivel de correlación, de r=0.916. Lo mismo ocurre con las dos variantes de la escala EPOA obtenidas en este segmento, pues correlacionan entre sí a un nivel de $r=0.913$ (ver figura 4).

Figura 4. Correlación de EPOAa1 y EPOAa2, segmento A

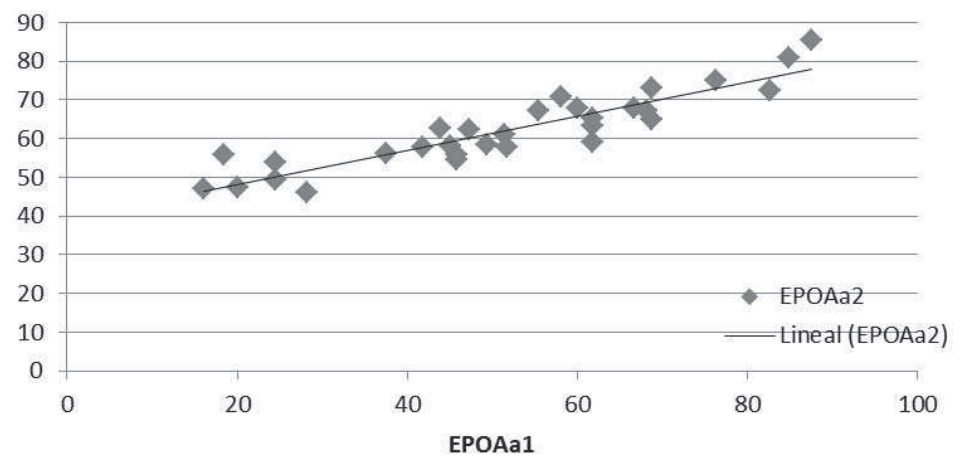

Los datos obtenidos en la variante EPOAa1 correlacionan positivamente respecto a la escala SIOPS, como puede observarse en la Figura 5. Lo hacen con un coeficiente de $r=0.784$, que queda ligeramente por debajo del $r=0.79$ en base al cual Treiman argumenta que es posible defender la homogeneidad de la escala a nivel internacional en las sociedades modernas complejas, y que considera suficiente para rechazar las críticas sobre la posible influencia del contexto cultural local en la escala (Bergman y Joye 2001). Por su parte, la variante EPOAa2 presenta una mayor dispersión y por lo tanto una menor correlación, de $\mathrm{r}=0.671$, por el efecto esperado de la introducción de las ocupaciones con prestigio negativo.

Figura 5. Correlaciones de las escalas EPOA del segmento A respecto a SIOPS

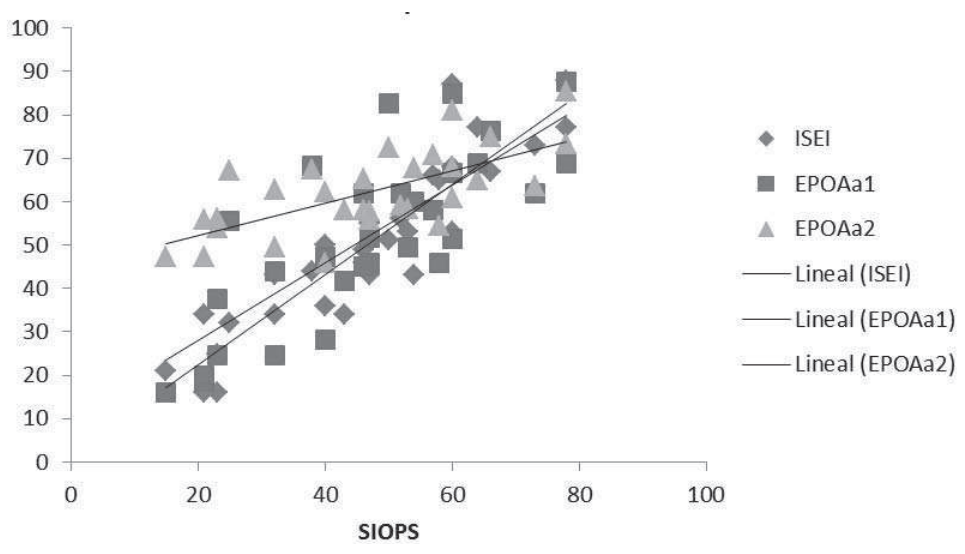

EMPIRIA. Revista de Metodología de Ciencias Sociales. N. ${ }^{\circ}$ 26, julio-diciembre, 2013, pp. 117-148. ISSN: 1139-5737, DOI: 10.5944/empiria.26.7155 
Ambas escalas, EPOA a1 y a2, también correlacionan positivamente con el ISEI, aunque en proporciones menores, de $\mathrm{r}=0.765$ en el caso de EPOAa1, que presenta un comportamiento más similar al que tiene SIOPS con respecto a ISEI; y de $\mathrm{r}=0.668$ en el de EPOAa2, que nuevamente se presenta más dispersa, tal como se ilustra en la Figura 6.

Figura 6. Correlaciones de las escalas EPOA del segmento A respecto a ISEI

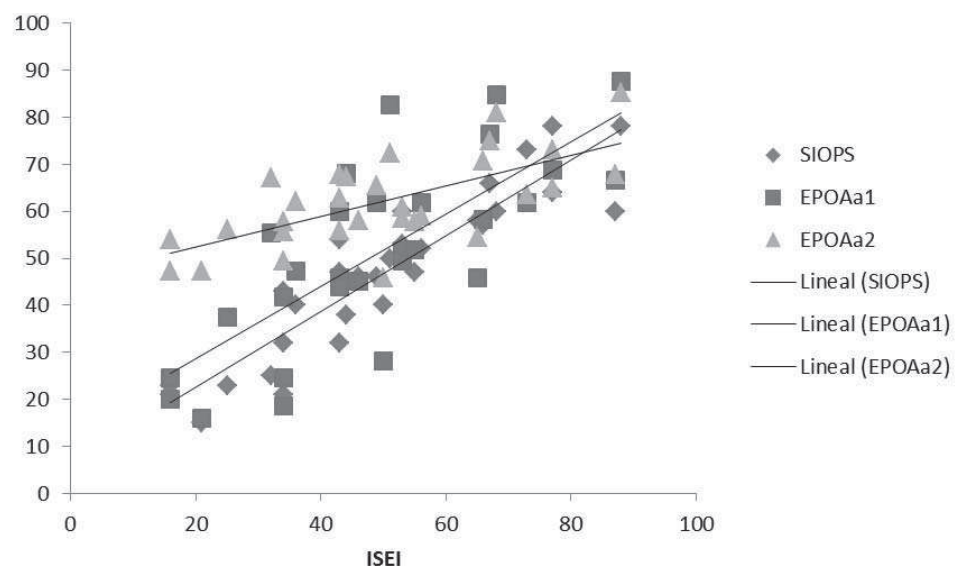

Una mirada más detallada al comportamiento de las ocupaciones comparando las diferentes escalas a partir de la figura 7, permite apreciar las fuentes del desajuste, que en nuestra opinión y en el contexto estudiado, se vuelven muy significativas.

En principio, la escala EPOAa2, obtenida por el método cuantitativo, se muestra más desfasada en todos los rangos, lo que podría deberse a que los informantes evitaron en general aproximarse al límite inferior de la escala de base 100 utilizada para puntuar las ocupaciones. De hecho, el albañil, la ocupación más baja, fue evaluado con 47 puntos.

Respecto a SIOPS, el ISEI presenta un claro desfase en determinadas ocupaciones cuya apreciación es más elevada, como ocurre con el banquero, el diputado o el gran empresario. Sin embargo, EPOAa1 muestra que las ocupaciones relativas a la especialización productiva de la localidad tienden a presentar niveles más altos de prestigio ocupacional que estas actividades, internacionalmente reconocidas como de un alto estatus socioeconómico.

Se puede deducir que nuestros informantes interpretan que tener un negocio, ya sea de manufactura, comercio o servicios, es una ocupación con más prestigio que profesiones como las de abogado, profesor o enfermera; inclusive el vendedor ambulante o en tianguis (mercados semifijos) puntúa ligeramente mejor en ambas versiones de la EPOA que en la SIOPS y el ISEI. Las ocupaciones diferentes al empresariado, pero relacionadas con la industria de la ropa, también son valuadas más alto que en las EIE, como es el caso de los cortadores o los operarios de máquinas de coser. Sin embargo, en las ocupaciones de menor 
calificación, los rangos tienden a no presentar variaciones significativas. Esto demuestra que la propia comunidad construye sus formas de prestigio, independientemente de las dictadas por las sociedades modernas.

Figura 7. Gráfica comparativa de puntuaciones EIE-EPOA para las ocupaciones del segmento A. Estudio sobre percepción del prestigio ocupacional en Uriangato, 2011

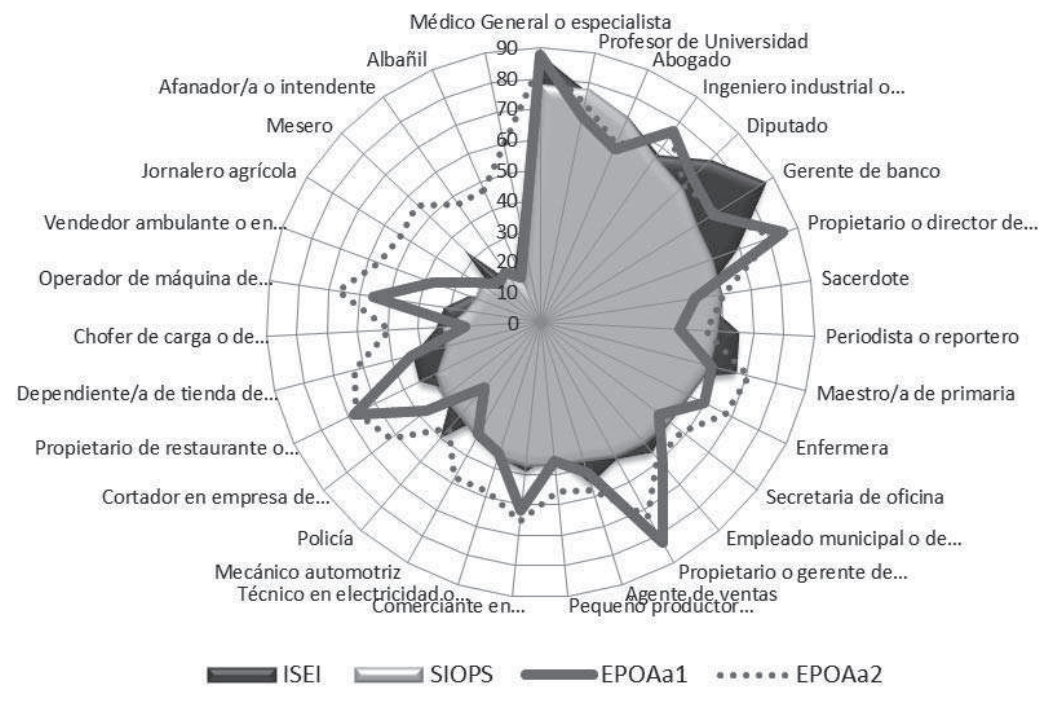

Las implicaciones en la determinación de la jerarquía de prestigio ocupacional son notables, como se aprecia en la Tabla 6. Para efectos de contraste consideramos conveniente señalar el comportamiento del ranking de ocupaciones según la escala EPOAa1, obtenida por la técnica cualitativa, frente al de SIOPS, donde sólo siete de las 30 ocupaciones mantienen su estatus: el médico en la parte alta; el ingeniero industrial o mecánico y el agente de ventas; y todos los de la parte baja de la tabla, es decir el jornalero agrícola, el trabajador de limpieza, el mesero y el albañil.

Nueve ocupaciones ven incrementado su nivel de prestigio, algunas de manera moderada como el dependiente de tienda de ropa, el vendedor ambulante, el cortador en empresa textil o el empleado municipal. Otras ocupaciones tienen un ascenso mucho más pronunciado, pues el propietario de empresa grande sube cuatro lugares, el comerciante seis, el operador de máquina de coser nueve, el propietario de pequeña empresa $11 \mathrm{y}$ el de restaurante 15 .

Las 14 ocupaciones restantes descienden en el ranking. Algunas lo hacen discretamente, como el diputado, el gerente de banco o la enfermera (un nivel); otras moderadamente, como los profesores de universidad y primaria (tres niveles), el policía y el productor agropecuario (cuatro puestos) o la secretaria con cinco; y algunas de manera pronunciada, como el abogado que se desplaza seis niveles hacia abajo, el sacerdote ocho y el periodista diez. 
En el caso del ranking de EPOAa2, suben de rango 14 ocupaciones, destacando el propietario de restaurante en 13 posiciones y el operario de máquina de coser en 15; descienden 13 actividades, más notablemente el abogado y el periodista, que pierden 10 y 16 lugares respectivamente, aunque el policía desciende al fondo de la escala; y sólo tres conservan el sitio, a saber, el médico, el banquero y el afanador.

Tabla 6. Ranking de ocupaciones según prestigio ocupacional según escalas EPOA segmento A, SIOPS e ISEI. Estudio sobre percepción del prestigio ocupacional en Uriangato, 2011

\begin{tabular}{lcccc}
\hline CMO OCUPACIÓN & RKEPOAa1 RKEPOAa2 RKSIOPS RKISEI \\
\hline 1130 Médico General o especialista & 1 & 1 & 1 & 1 \\
1300 Propietario o director empresa grande & 2 & 2 & 6 & 6 \\
1160 Propietario o gerente pequeña empresa & 3 & 5 & 14 & 14 \\
1104 Ingeniero industrial o mecánico & 4 & 3 & 4 & 7 \\
2100 Profesor de Universidad & 5 & 4 & 2 & 3 \\
2121 Diputado & 6 & 12 & 5 & 4 \\
2111 Propietario de restaurante & 7 & 9 & 22 & 18 \\
1180 Gerente de banco & 8 & 7 & 7 & 2 \\
1401 Abogado & 9 & 13 & 3 & 5 \\
1330 Empleado municipal o de gobierno & 10 & 17 & 13 & 10 \\
1220 Comerciante en establecimiento & 11 & 11 & 17 & 16 \\
6200 Enfermera & 12 & 8 & 11 & 19 \\
6290 Maestro/a de primaria & 13 & 6 & 10 & 8 \\
2124 Operador de máquina de coser & 14 & 10 & 25 & 26 \\
7130 Agente de ventas & 15 & 21 & 15 & 11 \\
4120 Sacerdote & 16 & 16 & 8 & 12 \\
7100 Secretaria de oficina & 17 & 18 & 12 & 13 \\
1205 Cortador en empresa de confección & 18 & 15 & 20 & 22 \\
5245 Periodista o reportero & 19 & 25 & 9 & 9 \\
5322 Pequeño productor agropecuario & 20 & 23 & 16 & 20 \\
8301 Técnico en electricidad o electrónica & 21 & 19 & 18 & 17 \\
2127 Dependiente/a de tienda de ropa & 22 & 14 & 23 & 21 \\
5520 Mecánico automotriz & 23 & 20 & 19 & 23 \\
7111 Vendedor ambulante o en tianguis & 24 & 22 & 26 & 27 \\
5322 Policía & 25 & 30 & 21 & 15 \\
4109 Chofer de carga o de pasajeros & 26 & 27 & 24 & 24 \\
7201 Jornalero agrícola & 27 & 26 & 27 & 29 \\
8124 Afanador/a o intendente & 28 & 28 & 28 & 30 \\
8101 Mesero & 29 & 24 & 29 & 25 \\
5260 Albañil & 30 & 29 & 30 & 28 \\
\hline
\end{tabular}

EMPIRIA. Revista de Metodología de Ciencias Sociales. N. ${ }^{\circ}$ 26, julio-diciembre, 2013, pp. 117-148. ISSN: 1139-5737, DOI: 10.5944/empiria.26.7155 


\section{Análisis del Segmento B}

En la tabla 7 se registran las puntuaciones obtenidas a través del segundo generador de posiciones, cuyo listado de 30 ocupaciones incluye, además de las estándar, otras con prestigio considerado como negativo y tres no contempladas en la CMO y no puntuadas en las EIE. Cabe recordar que en la segunda medición de este segmento se utilizó un rango de valoración distinto, que incluyó una escala entre -100 y 100, para observar el posible nivel de desprestigio que los informantes adjudicaran a determinadas ocupaciones.

Tabla 7. Puntuaciones de prestigio de las ocupaciones en el segmento B. Estudio sobre percepción del prestigio ocupacional en Uriangato, 2011

\begin{tabular}{|c|c|c|c|c|c|}
\hline $\mathrm{CMO}$ & OCUPACIÓN & EPOAb1 & EPOAb2 & SIOPS & ISEI \\
\hline 1160 & Abogado & 75 & 74 & 73 & 73 \\
\hline 8150 & Adivino, brujo o curandero & 20 & -9 & 37 & 43 \\
\hline 8124 & Afanador o trabajador de limpieza & 35 & 51 & 21 & 16 \\
\hline 5260 & Albañil & 30 & 52 & 15 & 21 \\
\hline N/A & Ama de casa & 41 & 68 & N/D & N/D \\
\hline 5240 & Ayudante de tejido o bordado & 40 & 54 & 18 & 20 \\
\hline 7100 & Comerciante en establecimiento & 71 & 68 & 46 & 49 \\
\hline 5322 & Cortador en empresa textil y de confección & 66 & 60 & 40 & 36 \\
\hline 7111 & Dependiente/a de tienda de ropa & 50 & 55 & 32 & 43 \\
\hline 6290 & Empleado municipal o de gobierno & 66 & 78 & 52 & 56 \\
\hline 7130 & Gestor o intermediario comercial (coyote) & 12 & 10 & 42 & 55 \\
\hline N/A & Jubilado o retirado & 31 & 58 & N/D & N/D \\
\hline 5222 & Maquilador de ropa a domicilio & 54 & 57 & 34 & 36 \\
\hline 1130 & Médico general o especialista & 93 & 93 & 78 & 88 \\
\hline 5322 & operario/a industrial de máquina de coser & 53 & 59 & 25 & 32 \\
\hline 8130 & Peluquero o estilista & 37 & 54 & 32 & 29 \\
\hline 8301 & Policía & 33 & 35 & 40 & 50 \\
\hline 8190 & Prestamista o agiotista & 34 & 25 & 15 & 40 \\
\hline 4120 & Productor agropecuario o ganadero & 52 & 68 & 47 & 43 \\
\hline 1330 & Profesor de primaria & 71 & 75 & 57 & 66 \\
\hline 7131 & Propietario arrendador de locales o fincas & 69 & 73 & 37 & 30 \\
\hline 2124 & Propietario de empresa & 84 & 84 & 50 & 51 \\
\hline 2127 & Propietario de restaurante/negocio comida & 71 & 80 & 38 & 44 \\
\hline 7130 & Proveedor de maquinaria o materia prima & 64 & 75 & 47 & 55 \\
\hline 1180 & Sacerdote & 71 & 85 & 60 & 53 \\
\hline 5246 & Técnico o mecánico de maquinaria industrial & 68 & 71 & 42 & 33 \\
\hline N/A & Trabajador no asalariado en negocio familiar & 63 & 72 & N/D & N/D \\
\hline 5229 & Transportista & 35 & 55 & 52 & 51 \\
\hline 7201 & Vendedor ambulante o en tianguis & 38 & 46 & 23 & 25 \\
\hline 7201 & Vendedor de mercancía pirata & 27 & -7 & 12 & 28 \\
\hline
\end{tabular}

EMPIRIA. Revista de Metodología de Ciencias Sociales. N. ${ }^{\circ}$ 26, julio-diciembre, 2013, pp. 117-148. ISSN: 1139-5737, DOI: 10.5944/empiria.26.7155 
La introducción deliberada de estos elementos de contraste tuvo como propósito observar hasta dónde se altera la congruencia de la jerarquía obtenida en el segmento A, específicamente en el caso de 16 ocupaciones tradicionales y localmente pertinentes de diferentes niveles de las EIE, que como mecanismo extra de validación mantuvimos en ambos cuestionarios, conservando su denominación o adquiriendo una equivalente.

Por efecto de estos mismos elementos, ninguna de las dos variantes obtenidas, EPOAb1 y EPOAb2, puede ser sujeta de un cálculo de correlación respecto a las EIE. En cambio, es posible verificar que entre ellas hay una correlación de $\mathrm{r}=0.8425$, lo que confirma un nivel aceptable de consistencia en las opiniones de los informantes en ambos esquemas de puntuación, no obstante la importante dispersión ocasionada por las dos puntuaciones de rango negativo que se obtuvieron, como puede apreciarse en la figura 8 .

Figura 8. Correlación de EPOAb1 y EPOAb2, segmento B

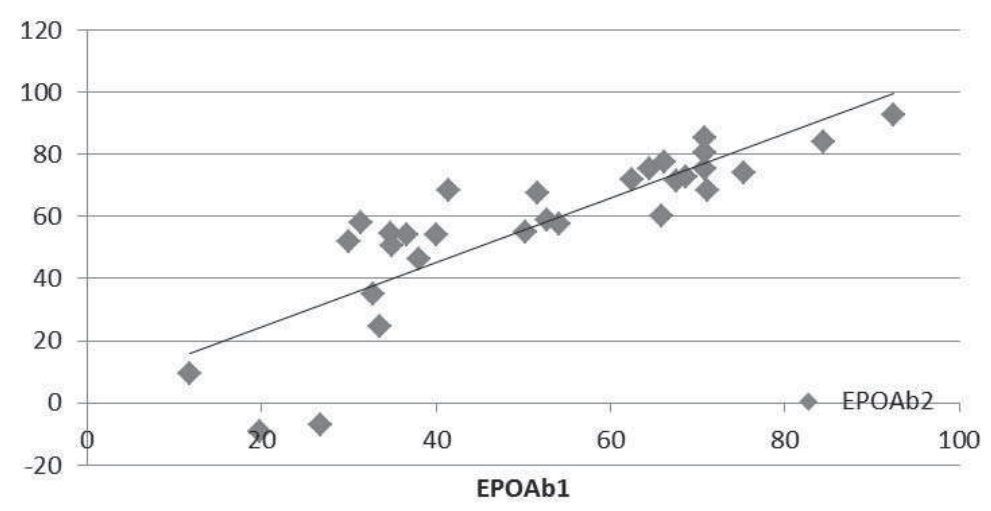

Tal como se esperaba, las ocupaciones deliberadamente introducidas con este propósito recibieron la mayor parte de los 20 puntajes negativos que en total se asignaron por los informantes: el adivino o brujo y el vendedor de mercancía pirata, cinco cada uno; el gestor o intermediario cuatro; y el prestamista dos. También fueron puntuados negativamente en dos ocasiones el policía, y en una el ama de casa y el jubilado.

En general, los informantes en este segmento evitaron valorar las ocupaciones por debajo de cero. Sin embargo, el uso de estas puntuaciones negativas parece tener un efecto en la apreciación positiva de ciertas ocupaciones, como la de sacerdote o los profesores, que en esta versión recuperan lugares en comparación con el segmento A.

El comportamiento de las ocupaciones en los ranking es también un indicador de la dimensión del nivel de desprestigio de algunas de ellas (ver tabla 8) en el marco cultural local observado. Por ejemplo, la percepción del gestor o intermediario comercial (Broker), denominado coloquialmente en México como coyote, tiene una connotación fuertemente negativa ${ }^{2}$, que explicaría el por qué, siendo

${ }^{2}$ Debido a que esta actividad puede tener un componente de ilegalidad, ya que su función se 
una ocupación por encima de media tabla en las EIE, en la EPOA se ubica en los últimos sitios. Lo mismo ocurre con el policía.

Tabla 8. Ranking de ocupaciones según prestigio ocupacional según escalas EPOA segmento B, SIOPS e ISEI. Estudio sobre percepción del prestigio ocupacional en Uriangato, 2011

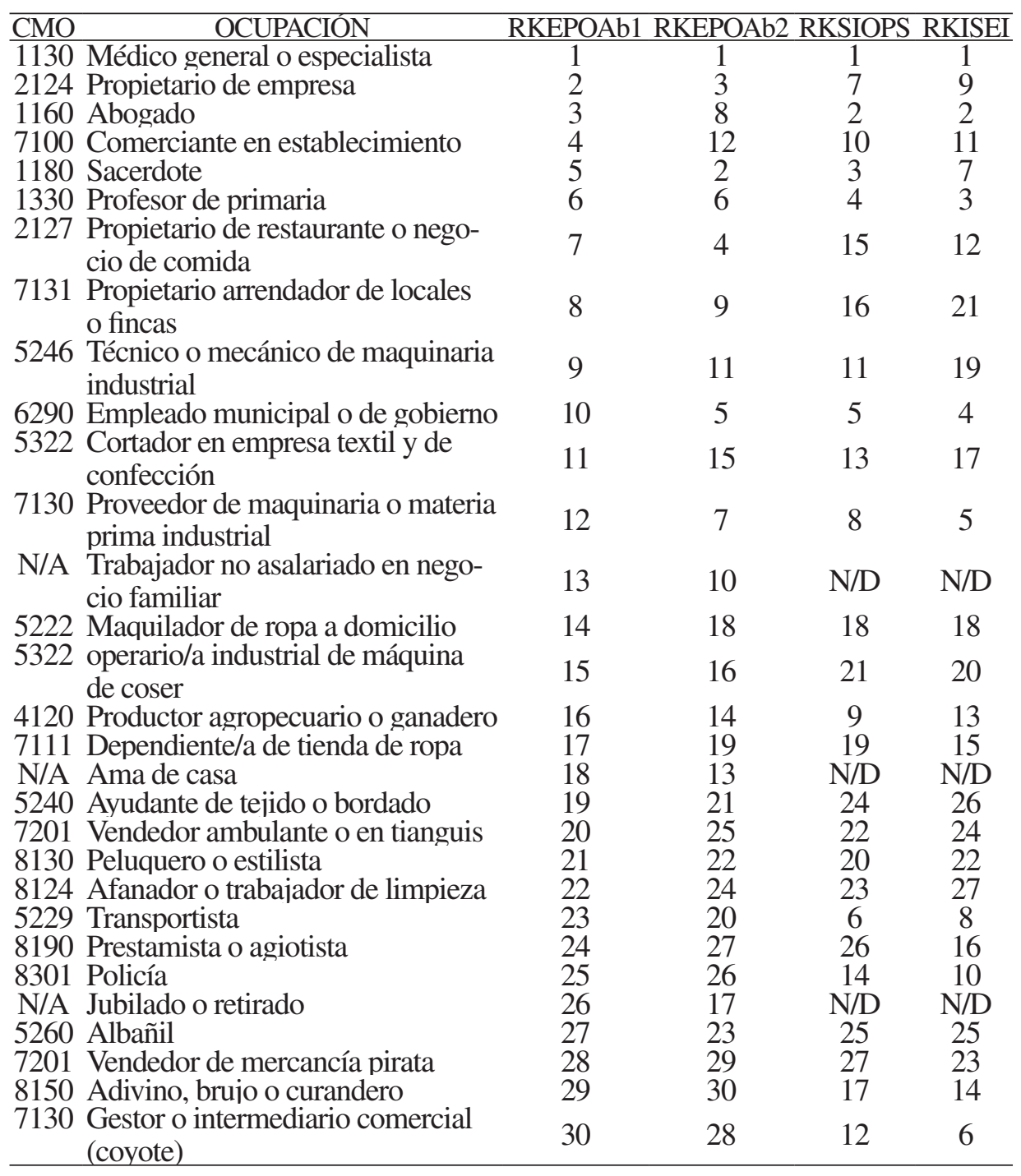

orienta a solucionar trámites de manera simplificada o sin cubrir todos los requisitos establecidos, en ocasiones por la vía de la corrupción a través de contactos personales (capital social negativo). 
Por el contrario, y tal como ocurrió en el segmento A, las ocupaciones relacionadas a la industria de la ropa y a otras de alta concentración en Uriangato, como el comercio y los servicios, tienden a ser mejor valoradas que en el estándar internacional.

Del análisis de los ranking también se desprende que los informantes adjudican niveles de prestigio significativos a las ocupaciones no incluidas en la CMO ni en las EIE, en este caso los trabajadores no asalariados, las amas de casa y, en menor grado, los jubilados. Se trata de ocupaciones socialmente valoradas, cuya exclusión de un análisis basado en jerarquías ocupacionales en este contexto, podría ser fuente de sesgos importantes.

\section{Análisis de las ocupaciones comunes en ambos segmentos.}

La tabla 9 recupera las puntuaciones de las 16 ocupaciones que se mantuvieron constantes en los dos segmentos de nuestro estudio. A partir de ellas, se puede tener una idea clara del nivel en que cada procedimiento de jerarquización utilizado en el proceso de adaptación/validación de las EIE en el contexto atípico local, impactaría en un pequeño listado de ocupaciones estándar, similar a un Generador de posiciones tradicional, cuyos datos podrían compararse aceptablemente con los obtenidos en estudios realizados en contextos económicos, productivos o culturales diferentes.

Tabla 9. Puntuación de 16 ocupaciones comunes en los segmentos A y B, según EPOA. Estudio sobre percepción del prestigio ocupacional en Uriangato, 2011

\begin{tabular}{lcccc}
\hline \multicolumn{1}{c}{ OCUPACIÓN } & EPOAa1 & EPOAa2 & EPOAb1 & EPOAb2 \\
\hline Médico General o especialista & 88 & 85 & 93 & 93 \\
Propietario o gerente de pequeña empresa & 83 & 72 & 84 & 84 \\
Propietario de restaurante/negocio de comida & 68 & 67 & 71 & 80 \\
Comerciante en establecimiento & 62 & 65 & 71 & 68 \\
Abogado & 62 & 63 & 75 & 74 \\
Empleado municipal o de gobierno & 62 & 59 & 66 & 78 \\
Maestro/a de primaria & 58 & 71 & 71 & 75 \\
Operador de máquina de coser & 55 & 67 & 53 & 59 \\
Sacerdote & 52 & 61 & 71 & 85 \\
Cortador en empresa de confección & 47 & 62 & 66 & 60 \\
Pequeño productor agropecuario & 46 & 56 & 52 & 43 \\
Dependiente/a de tienda de ropa & 44 & 63 & 50 & 55 \\
Vendedor ambulante o en tianguis & 38 & 56 & 38 & 46 \\
Policía & 28 & 46 & 33 & 35 \\
Afanador/a o intendente & 20 & 47 & 35 & 51 \\
Albañil & 16 & 47 & 30 & 52 \\
\hline
\end{tabular}


Los datos a este nivel confirman la congruencia entre las opiniones de los informantes al interior de cada segmento, con independencia del método de puntuación que se utilice. Sin embargo, como se aprecia en la tabla 10, los datos obtenidos a través de la variante cualitativa por el método de ordenación jerárquica de tarjetas y expresados en EPOAa1 y EPOAb1, correlacionan mejor entre sí, de forma muy cercana a como lo hacen SIOPS e ISEI. Por su parte, los que se obtuvieron a través del método de puntuación numérica, también correlacionan entre sí de manera positiva, aunque más débilmente ( $\mathrm{r}=0.7825)$.

Tabla 10. Coeficientes de correlación para 16 ocupaciones comunes en los segmentos A y B, según tipo de escala EPOA y EIE. Estudio sobre percepción del prestigio ocupacional en Uriangato, 2011

\begin{tabular}{lccccc}
\hline & EPOAa2 & EPOAb1 & EPOAb2 & SIOPS & ISEI \\
\hline EPOAa1 & 0.9143 & 0.9443 & 0.8255 & 0.7305 & 0.7468 \\
EPOAa2 & & 0.8843 & 0.7825 & 0.6385 & 0.6985 \\
EPOAb1 & & & 0.8982 & 0.8238 & 0.7849 \\
EPOAb2 & & & & 0.6885 & 0.6621 \\
SIOPS & & & & & 0.9514 \\
\hline
\end{tabular}

La mayor congruencia de nuestros datos con las dos EIE se obtiene a través de la escala EPOAb1, seguida por EPOAa1, ambas obtenidas por el método cualitativo del pile sort. Los valores obtenidos por los mecanismos de asignación libre de puntos, aparecen con correlaciones positivas, pero mucho más débiles, respecto a las EIE.

\section{UNA MUESTRA DE OCUPACIONES LOCALMENTE PERTINENTE, PARA UN GENERADOR DE POSICIONES INTERNACIONALMENTE COMPATIBLE}

Con el fin de disponer de un instrumento preciso, se procedió a construir un listado común que incluyera todas las ocupaciones comparables con las EIE utilizadas en ambos instrumentos, y a catalogarlas conforme una escala única que toma como base las puntuaciones de las escalas EPOAa1 y EPOAb1. En el caso de las ocupaciones duplicadas se mantuvo la puntuación que obtuvieron en el primer instrumento, en el que sí fue posible observar las correlaciones mantenidas con las EIE.

A partir de este listado general se realizó una selección de 20 ocupaciones que consideramos congruentes con la especialización de la estructura del mercado de trabajo de la localidad, y que se presenta en la tabla 11. Las ocupaciones están distribuidas equilibradamente en una estructura piramidal, congruente con la idea de reflejar la distribución desigual del prestigio al momento de estimar el Capital Social. El rango conforme a EPOA va desde 88 puntos, que corresponden al médico general o especialista, hasta los 16 del albañil (Ver figura 9). 
Tabla 11. Listado final de ocupaciones para la estimación del Capital Social en un contexto atípico, conforme a la EPOE y las EIE

\begin{tabular}{lccc}
\hline CMO OCUPACIÓN & EPOA & SIOPS & ISEI \\
\hline 1130 Médico General o especialista & 88 & 78 & 88 \\
2124 Propietario o gerente pequeña empresa & 83 & 50 & 51 \\
1104 Ingeniero industrial o mecánico & 76 & 66 & 67 \\
2127 Propietario de restaurante & 68 & 38 & 44 \\
7130 Proveedor de maquinaria o materia prima industrial & 64 & 47 & 55 \\
7100 Comerciante en establecimiento & 62 & 46 & 49 \\
5322 Operador de máquina de coser & 55 & 25 & 32 \\
5222 Maquilador de ropa a domicilio & 54 & 34 & 36 \\
7130 Agente de ventas & 52 & 47 & 55 \\
4120 Pequeño productor agropecuario & 46 & 47 & 43 \\
5322 Cortador en empresa de confección & 47 & 40 & 36 \\
7111 Dependiente/a de tienda de ropa & 44 & 32 & 43 \\
5240 Ayudante de tejido o bordado & 40 & 18 & 20 \\
7201 Vendedor ambulante o en tianguis & 38 & 23 & 25 \\
8190 Prestamista o agiotista & 34 & 15 & 40 \\
7201 Vendedor de mercancía pirata & 27 & 12 & 28 \\
4109 Jornalero agrícola & 25 & 23 & 16 \\
8124 Afanador/a o intendente & 20 & 21 & 16 \\
8101 Mesero & 18 & 21 & 34 \\
5260 Albañil & 16 & 15 & 21 \\
\hline
\end{tabular}

Figura 9. Distribución jerárquica de las puntuaciones de prestigio ocupacional del listado final conforme a EPOA

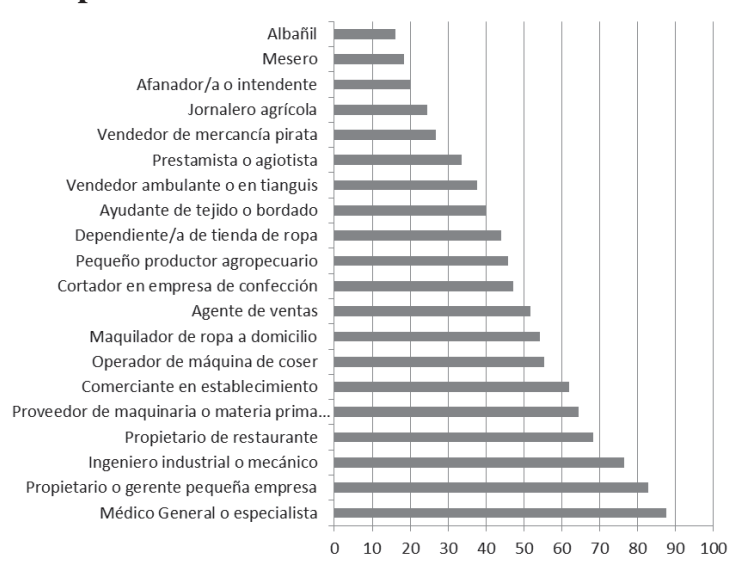

EMPIRIA. Revista de Metodología de Ciencias Sociales. N. ${ }^{\circ}$ 26, julio-diciembre, 2013, pp. 117-148. ISSN: 1139-5737, DOI: 10.5944/empiria.26.7155 
Al calcular el coeficiente de correlación de los puntajes EPOA de este listado final de 20 ocupaciones respecto a las EIE, se encontró una consistencia elevada, pues respecto a SIOPS es de $\mathrm{r}=0.8624$ y respecto a ISEI, de $\mathrm{r}=0.8310$.

Estos indicadores son lo suficientemente fuertes para afirmar que este listado, al ser incluido en un Generador de Posiciones para estimar el Capital Social en este contexto atípico, no sólo otorgará mayor precisión que la que podría haberse obtenido mediante la utilización de las EIE, sino que al correlacionar fuertemente con los valores de aquellas, también permitirá mantener la comparabilidad de los resultados obtenidos, con los de otros estudios realizados en regiones o contextos diferentes, siempre que tengan como base a las EIE o a diversas adaptaciones congruentes con éstas.

\section{CONCLUSIONES}

Nuestro estudio demuestra que la percepción del prestigio ocupacional en esta localidad atípica, efectivamente difiere de la que se tiene en contextos tradicionales y especialmente en las sociedades modernas complejas, en las cuales las escalas internacionalmente estandarizadas han sido ampliamente utilizadas en las estimaciones de capital social desde la óptica posicional.

En concreto, demostramos que las ocupaciones representativas de la configuración especializada del mercado de trabajo, de la actividad sustantiva de la localidad, se evalúan en términos más elevados que otras actividades normalmente ubicadas en rangos más altos de prestigio o estatus socioeconómico en contextos típicos, lo cual exige metodológicamente que las distintas comunidades que se estudian se interpreten de manera diferenciada. Una aportación, en este sentido sería la valoración de las especificidades locales, sin menoscabo de la posibilidad de generalizar.

Hemos mostrado también que la construcción de una escala localmente adaptada, no sólo resulta consistente con las Escalas Internacionalmente Estandarizadas, sino que incluso es capaz de correlacionar mejor con ellas cuando se incluyen ocupaciones pertinentes al contexto local, aún si su prestigio parece ser negativo.

El hallazgo más relevante, en nuestro juicio, consiste en que la utilización de una técnica de carácter cualitativo, el método de ordenación de tarjetas, reflejó con una mayor consistencia la percepción local en torno a la estructura ocupacional y a la manera en que ésta se estratifica en el contexto específico y los rasgos culturales de la comunidad.

Al ser obtenido por este método, el listado final de ocupaciones que incluiremos en futuros Generadores de Posiciones, no sólo permitirá estimar adecuadamente el Capital Social de esta localidad, sino también mantener la comparabilidad con estudios realizados en otras latitudes y contextos -siempre que se basen en las Escalas Internacionalmente Estandarizadas- incluyendo aquellos no considerados como atípicos. 
Creemos que el modelo cultural en torno a la estructuración social del trabajo en Uriangato nos abre avenidas para comprender de qué modo influye en ella el Capital Social. Es un trabajo aún en marcha.

\section{BIBLIOGRAFÍA}

BAROZET, E. (2007): La variable ocupación en los estudios de estratificación social. Disponible en http://www.desigualdades.cl/wp-content/uploads/2009/05/ocupacion. pdf [consulta 20-06-2011].

BEKKERS, R., VÖLKER, B., VAN DER GAAG, M., Y FLAP, H. (2008): «Social Networks of participants in voluntary associations», en Social Capital: An international Research Program, Oxford, New York, Oxford University Press, pp. 185-205.

BERGMAN, M. M., y JOYE, D. (2001): «Comparing Social Stratification Schemas: CAMSIS, CSP-CH, Goldthorpe, ISCO-88, Treiman, and Wright», Cambridge studies in social research, 9, 1-37.

BIAN, Y. (2008): «The formation of Social Capital among chinese Urbanites: Theorethical Explanation and empirical evidence», en Social capital: An international Research Program, Oxford, New York, Oxford University Press, pp. 82-104.

BORGATTI, S.P., JONES, C. Y EVERETT, M. (1998): «Network Measures of Social Capital», Connections, 21 (2): 27-36. INSNA.

BRUNIE, A. (2009): «Meaningful distinctions within a concept: Relational, collective, and generalized social capital», Social science research, 38(2), pp. 251-265.

BURT, R. S. (2000): «The network structure of social capital», Research in Organizational Behavior, 22, pp. 345-423.

CARABAÑA, J., Y GÓMEZ, C. (1996): Escalas de prestigio profesional, Madrid, Centro de Investigaciones Sociológicas.

CHEN, W. Y TAN, J. (2009): «Understanding Transnational Entrepreneurship Through a Network Lens: Theoretical and Methodological Considerations», Entrepreneurship Theory and Practice, (September 2009), pp. 1079-1091.

DE LA GARZA, E. (2010): Hacia un concepto ampliado de trabajo. Del concepto clásico al no clásico. Barcelona, Anthropos. Universidad Autónoma Metropolitana.

ENNS, S., MALINICK, T., Y MATTHEWS, R. (2008): «It's Not Only Who You Know, It's also Where They Are: Using the position Generator to Investigate the Structure of Access to Embedded Resources» en Social Capital. An International Research Program, Oxford, New York, Oxford university Press, pp. 255-281.

FLAP, H., Y BOXMAN, E. (2001): «Getting started: The influence of social capital on the start of the occupational career» en Social Capital: Theory and Research, New York, Aldine de Gruyter, pp. 159-181.

FLAP, H., Y VÖLKER, B. (2008): «Social, Cultural, and Economic Capital and Job Attainment: The Position Generator as a Measure of Cultural and Economic Resources» en Social Capital. An International Research Program, Oxford, New York, Oxford University Press, pp. 65-80.

FULKERSON, G. M., Y THOMPSON, G. H. (2008): «The evolution of a contested concept: A meta-analysis of social capital definitions and trends (1988-2006)», Sociological Inquiry, 78(4), pp. 536-557. 
GANZEBOOM, H. B. G., Y TREIMAN, D.,J. (1996): «Internationally comparable measures of occupational status for the 1988 international standard classification of occupations», Social Science Research, 25, pp. 201-239.

GARCÍA MACÍAS, A. (2011): «Desarrollo local en contextos de industrialización atípica. Análisis de tres casos exitosos en el centro occidente de México», ponencia en $V$ coloquio de Investigación Nacional e Internacional de Cuerpos Académicos y ler Workshop de la PYME y desarrollo de lo local. México, Universidad Autónoma de Aguascalientes, Junio 23-24, 2011.

HUBER, F. (2009): «Social Capital of Economic Clusters: Towards a Network-Based Conception of Social Resources», Tijdschrift voor Economische en Sociale Geografie, Vol. 100, No. 2, pp. 160-170.

ILO (2011): ISCO. International Standard Classification of occupations, disponible en http://www.ilo.org/public/english/bureau/stat/isco/index.htm [consulta 28-06-2011]

INEGI (2009a): Clasificación Mexicana de Ocupaciones, disponible en http://www. inegi.org.mx/est/contenidos/Proyectos/encuestas/hogares/regulares/enoe/Default. aspx [consulta 28-06-2011].

INEGI (2009/b): Censos Económicos 2009. Resultados definitivos. Disponible en http:// www.inegi.org.mx/est/contenidos/espanol/proyectos/censos/ce2009/default. asp? $\mathrm{s}=\mathrm{est} \& \mathrm{c}=14220$ [Consulta 21-06-2011].

INEGI (2010): Censo de población y Vivienda 2010, Resultados Definitivos, disponible en http://www.censo2010.org.mx/ [Consulta 15-06-2011].

LIN, N. (2001): Social capital: A theory of social structure and action, Cambridge, Cambridge University Press.

LIN, N. (2008): «A network theory of social capital» en The Handbook of Social Capital. Oxford University Press, Oxford, pp. 50-69.

LIN, N., Y AO, D. (2008): «The Invisible Hand of Social Capital: An Exploratory Study», en Social Capital. An International Research Program», Oxford, New York, Oxford University Press, pp. 107-132.

LIN, N., Y ERICKSON, B. H. (2008): «Theory, Measurement, and the Research Enterprise on Social Capital», en Social Capital: An International Research Program, Oxford, New York, Oxford University Press, pp. 1-24.

LIN, N., FU, Y., Y HSUNG, R. (2001): «The position generator: Measurement techniques for investigations of social capital», en Social Capital: Theory and Research, New York, Aldine de Gruyter, pp. 57-81.

LOZARES, C., MOLINA, J. L., GARCÍA MACÍAS, A., Y MAZA, O. (2011): «The measurement of social capital using the position generator in social contexts with an atypical occupational configuration», Paper en International Seminar: Personal Networks, Methods and Applications, Barcelona, Universidad Autónoma de Barcelona, Julio 6-8, 2011.

MAZA, O., GARCÍA MACÍAS, A., Y ORTEGA, G. (2011): «Análisis de casos exitosos de comunidades centradas en el desarrollo de PYMES», Ponencia en XXIV Congreso Internacional de Sociología de las Organizaciones, Guanajuato, México. Asociación Internacional de Sociología de las Organizaciones. Julio de 2011.

MOLINA, J.L. Y BERTRÁN, J.V. (2008): «Tipologías y clasificaciones», Periferia, Revista de Recerca y Formació en Antropología, Barcelona, Universidad Autónoma de Barcelona.

NEFFA, J.C., OLIVERA, M.L., PERSIA, J., Y TRUCCO, P. (2010): La crisis de la relación salarial: naturaleza y significado de la informalidad, los trabajos/empleos precarios y los no registrados, Buenos Aires, CEIL-PIETTE CONICET. 
PORTES, A. (2000): «The Two meanings of Social Capital», Sociological Forum, Vol. 15, No. 1 (Mar., 2000), pp. 1-12.

PUTNAM, R. (2000): Bowling Alone: The Collapse and Revival of American Community. Simon \& Schuster, New York.

TORCHE, F. Y VALENZUELA, E. (2011): «Trust and reciprocity: A theoretical distinction of the sources of social capital», European Journal of social Theory, 14 (2), pp. 181-198.

VAN DER GAAG, M. (2005): The measurement of individual Social Capital. Groningen, Rijksuniversiteit Groningen.

VAN DER GAAG, M., SNIJDERS, T.A.B., Y FLAP, H. (2008): «Position generator measures and their relationship to other social capital measures» en Social Capital: An International Research Program, Oxford, New York, Oxford University Press, pp. 27-48. 